\title{
Physiological and Anatomical Organization of Multiwhisker Response Interactions in the Barrel Cortex of Rats
}

\author{
Satoshi Shimegi, Takafumi Akasaki, Takehiko Ichikawa, and Hiromichi Sato \\ School of Health and Sport Sciences, Osaka University, Toyonaka, Osaka 560-0043, Japan
}

\begin{abstract}
To understand the physiological properties and anatomical organization of the spatiotemporal interaction of the responses to multiwhisker stimulation in neurons of the rat barrel cortex, single-unit recordings of 114 neurons were performed across all layers (layer II/III, $n=39$; IV, $n=33$; V/VI, $n=42$ ) of the posteromedial barrel subfield of the primary somatosensory cortex of anesthetized rats. Two neighboring principal and adjacent whiskers (PW and AW, respectively) in the same row were deflected rostrally or caudally at varying interstimulus intervals (ISIs). In $37 \%$ of the neurons, the response to the combined stimulus was significantly larger than the sum of the responses to stimulation of the individual whiskers. In instances in which response facilitation was observed, selectivity was noted for the combination $(75 \%)$ of the PW with a particular AW or for a particular direction (60\%) of whisker deflection. The direction bias of the responses to multiwhisker stimulation was well cor-
\end{abstract}

related with that of the sum of the responses to single whisker stimulation $(r=0.83 ; p<0.001)$. The pattern and magnitude of the response interaction in the neurons of the superficial layers were closely related to the location of the recorded cell in the barrel columns. Multiwhisker stimulation at short ISIs ( $\leq 3 \mathrm{msec})$ evoked prominent response facilitation in cells located close to the border between two columns ( $p<0.05$, one-way ANOVA), where two excitatory inputs were expected to arrive at the same time. Our results suggest that the spatiotemporal patterns of multiwhisker stimulation, such as whisker combination, direction of deflection, and timing, are expressed as different magnitudes of response interaction, which depends on the proximity of cells to home and adjacent barrel columns.

Key words: somatosensory system; barrel cortex; multiwhisker stimulation; response facilitation; stimulus specificity; spatiotemporal interaction
Mystacial whiskers of rodents are essential components of the somatosensory apparatus for the animals to perceive their surrounding environment (Vincent, 1912; Richter, 1957; Griffiths, 1960; Schiffman et al., 1970; Carvell and Simons, 1990). Although the whiskers are discrete tactile sensors that are scattered spatially on the face, animals require information about the spatiotemporally continuous representation of their surroundings in the behavioral context. From this point of view, it is natural to postulate that rats perceive three-dimensional stimulus features of the objects around them through a neural mechanism that enables integration of information derived from multiple whiskers.

In the posteromedial barrel subfield (PMBSF) of the primary somatosensory cortex (SI), neurons above, below, and within a barrel are postulated to form a columnar functional module for the processing of information, essentially from a corresponding single vibrissa (Simons and Woolsey, 1979; Chmielowska et al., 1986). However, there is also a convergence of information from the surrounding whiskers to a single column. Intracellular recording studies (Moore and Nelson, 1998; Z hu and Connors, 1999) have revealed that single neurons in the barrel cortex have wide receptive fields and integrate excitatory inputs from $>10$ whiskers.

In rodents, information about the surrounding space is obtained from simultaneous and sequential multiwhisker contacts with objects (Carvell and Simons, 1990). There is evidence that in sequential multiwhisker stimulation, the second whisker response could be strongly suppressed by the first whisker-evoked inhibition (Simons, 1985; Kleinfeld and Delaney, 1996; Goldreich et al., 1998). Moreover, Simons and his colleagues (Simons, 1983, 1985; Carvell and

Received Dec. 29, 1999; revised May 9, 2000; accepted May 24, 2000

This work was supported by Grant-in-Aid for Scientific Research (B) (08458268), Grant-in-Aid for Scientific Research on Priority Areas (B) (08279229, 09268221 10164230), and Grant-in-Aid for Encouragement of Young Scientists (09780060) from the Japanese Ministry of Education, Science, Sports, and Culture, and JSPSRFTF96L00201 from the Japan Society for the Promotion of Science. We thank Dr. Yumiko Yoshimura for helpful comments.

Correspondence should be addressed to Hiromichi Sato, School of Health and Sport Sciences, Osaka University, Machikaneyama, Toyonaka, Osaka 560-0043, Japan. E-mail: j61343@center.osaka-u.ac.jp.

Copyright (C) 2000 Society for Neuroscience $0270-6474 / 00 / 206241-08 \$ 15.00 / 0$
Simons, 1988) reported that the inhibitory interaction showed specificity for attributes of the stimulus, such as the interstimulus interval (ISI), the angular direction of whisker deflection, the sequence of deflections, and the particular combination of whiskers stimulated. Therefore, it has been proposed that the stimulusspecific inhibitory interaction of the responses to multiwhisker stimulation plays an important role in the processing of whisker information for a representation of the three-dimensional environment around an animal (Keller, 1995).

However, more recent studies reported new findings on the mechanisms of response integration in the PMBSF. First, multiwhisker stimulation has been reported to evoke a facilitatory interaction in the barrel cortex of anesthetized rats (Ghazanfar and Nicolelis, 1997; Shimegi et al., 1999). The ISI that can evoke response facilitation was well tuned to a narrow range (mean $\pm \mathrm{SD}$, $5.3 \pm 2.3 \mathrm{msec}$ ) (Shimegi et al., 1999). Second, in awake rats, sensory responses of neurons in the SI and the ventral posterior medial nucleus of the thalamus (VPM) are dynamically modulated depending on the behavioral states of animals (Fanselow and Nicolelis, 1999). These results suggest that the discrimination of the spatiotemporal sequence of stimulating different whiskers is probably achieved by not only inhibitory but also facilitatory interactions of the responses, and the interaction would be modulated according to the behavioral context.

In this report, we address the physiological properties of facilitatory interaction, particularly in relation to stimulus specificity, of multiwhisker responses in the barrel cortex and also the anatomical organization underlying these response interactions.

Details of the experimental methods used in this study have been published previously (Shimegi et al., 1999).

\section{MATERIALS AND METHODS}

Single-unit recordings were performed from the PMBSF of the SI of 53 Sprague Dawley rats weighing between 200 and $450 \mathrm{gm}$. All efforts were made to minimize the animal suffering and the number of animals used. The surgical procedures used were all in accordance with the National Institutes of Health guidelines for the care of experimental animals (National Institute of Health, Committee on Care and Use of Laboratory 
A

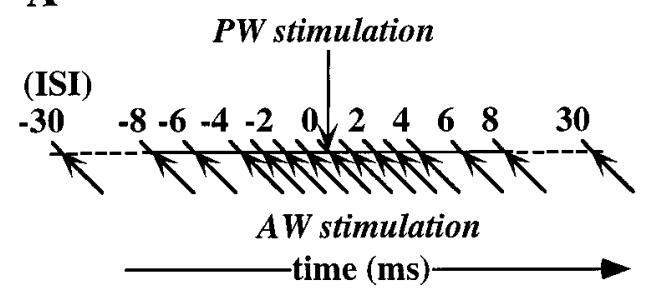

B

Combination $1 \quad$ Combination 2

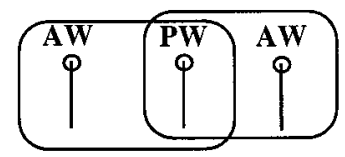

C

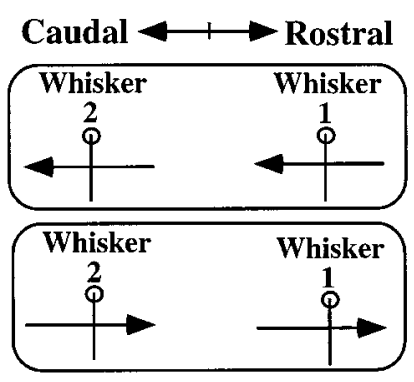

Figure 1. Schematic of the paradigm of combined whisker stimulation. $A$ The principal and adjacent whiskers ( $P W$ and $A W$, respectively) in the same row were briefly deflected either in the rostral or caudal direction at intervals of $0,1,2,3,4,6,8,10,12$, and $30 \mathrm{msec}$. The timing of whisker stimulation is indicated by arrows. The paired whiskers were deflected in the same direction under all stimulus conditions. $B$, Protocols to test the selectivity for specific combinations of whiskers. Response to combined stimulation of a PW and a rostral AW was compared with that of the PW and a caudal AW. $C$, Protocols to test the selectivity for direction of whisker deflection. A pair of whiskers was stimulated in two opposite directions (rostral or caudal) from the resting position.

Animals, 1985) and the guidelines of the Animal Care Committee of the Osaka University Medical School.

Preparation. The animals were anesthetized with urethane $(1.25 \mathrm{gm} / \mathrm{kg}$, i.p.). The local anesthetic lidocaine was given at pressure points and around the area of surgery. After the initial surgery, the animal was placed on a stereotaxic headholder. The depth of anesthesia was monitored throughout the duration of the experiment by testing for reflexes and monitoring the changes of heart rate in response to pinching of the tail. If the heart rate changed in response to pinching of the tail, more urethane was administered. Regular respiration ( $80-100$ breaths $/ \mathrm{min})$ and absence of spontaneous movements were ensured. The rectal temperature was maintained at $37-38^{\circ} \mathrm{C}$ by a thermostatically controlled heating pad.

Device for whisker stimulation. Whiskers were stimulated mechanically by probes attached to a galvanometer (Ito et al., 1979; Ito, 1985). Whiskers contralateral to the exposed barrel cortex were trimmed to a length of 15 $\mathrm{mm}$ and securely held with the wedge at the tip of the probe. The tip of the stimulating probe was positioned at a distance of $10 \mathrm{~mm}$ from the facial skin. The excursion of the tip of the probe was $1.1 \mathrm{~mm}$ over $10 \mathrm{msec}$, and the onset and offset velocity was $110 \mathrm{~mm} / \mathrm{sec}$ at the tip of the probe without a hold phase in either direction. This velocity was sufficient to elicit supramaximal responses in the SI neurons, as reported by Ito (Ito et al., 1979; Ito, 1985). The whisker was deflected rostrally or caudally from its natural position.

Whisker stimulation. The protocol used for whisker stimulation is illustrated in Figure 1. Basically, a pair of neighboring principal and adjacent whiskers (PW and AW, respectively) in the same row was deflected either simultaneously or successively at varying ISIs. For the sequential stimulation, the PW was stimulated before or after the AW at varying ISIs. Most units were tested with a full set of ISIs of $0,1,2,3,4,6,8,10,12$, and 30 msec (Fig. 1A). In some instances, longer ISIs of 30, 60, 100, 200, 300, and $400 \mathrm{msec}$ were also tested. In this paper, a set of data obtained using a full set of ISIs for a particular whisker combination is called a "case." When a cell remained stable long enough to allow testing with more than one set of ISIs, it was tested for a few different combinations of two whiskers (Fig. $1 B$ ) and/or different directions of whisker deflection (Fig. 1C). A set of data for two different combinations of three whiskers stimulated, that is, a PW and either a rostral or a caudal AW, is called a "sample." A set of data for two different directions of stimulation of the same whisker pair is also called a sample.

Electrophysiological recordings. A rectangular opening $(3 \times 4 \mathrm{~mm})$ was made above the left barrel cortex $(4-7 \mathrm{~mm}$ lateral to the midline and $0-4$ $\mathrm{mm}$ posterior to the bregma) to allow penetration of the recording electrodes. Single-pipette glass microelectrodes were used in this study to achieve the best isolation of a single-unit activity, as well as to obtain well localized dye marks of recording sites. The electrodes were filled with 0.5 M sodium acetate containing 4\% Pontamine Sky Blue (Tokyo Kasei, Tokyo, Japan) for histological identification of the recording sites. The resistance of the electrodes ranged from 13 to $22 \mathrm{M} \Omega$, as measured in situ. In most recordings, we could obtain well isolated single cells that exhibited unitary spikes with the same waveform, amplitude, and time course.

Once a single-unit activity was isolated, a PW was assessed qualitatively by manually deflecting the whiskers. Then, stimulating devices were set to stimulate the PW and AW in the same row. Recordings were restricted to cells whose PWs were a caudal group of whiskers including those in the D and $\mathrm{E}$ rows, $\gamma$ and $\delta$. The majority of cells was located around the barrel columns E1 and E2. $\gamma$ and $\delta$ whiskers were stimulated in combination with D1 or E1 whiskers.

Peristimulus time histograms (PSTHs) were constructed on-line during, mostly, 50 or, in some instances, 25 stimulations at a frequency of $0.5 \mathrm{~Hz}$ for each stimulus condition.

Analysis of responses. The response magnitude of a given cell was defined as the total number of spikes occurring between 5 and $37 \mathrm{msec}$ after the onset of whisker stimulation. However, when the late component of the response fell beyond this range, the time window was expanded long enough to include the late responses. The spontaneous firing rate was subtracted from the response magnitude. The whiskers whose stimulation elicited responses with the shortest latency or the greatest response magnitude were defined as PWs.

To quantitatively assess the response facilitation to combined whisker stimulation, we calculated the facilitation index (FI) (Shimegi et al., 1999), which was defined as the maximum number of spikes elicited by combined stimulation of two whiskers divided by the sum of the number of spikes evoked by single stimulation of each whisker. An FI value of $>1.0$ indicates a facilitatory interaction of the responses. We defined a response interaction with an FI $\geq 1.25$ as a significant facilitation, which will hereafter be referred to as "response facilitation." To confirm the validity of the criteria for facilitation, we calculated the variance of the sum of responses to single-whisker stimulation of PW and AW in three measurements of 50 stimulations in 38 cases showing FI $\geq 1.25$. Most ( $84 \%$, in 32 of 38 cases) of the responses to multiwhisker stimulation corresponding to $\mathrm{FI}=1.25$ were $\geq 1 \mathrm{SD}$ of the distribution of the sum of responses to single-whisker stimulation. Moreover, in 33 of 38 cases $(87 \%)$, the maximal response to multiwhisker stimulation for each case was greater than the confidence level ( $p=0.01$ ) of the distribution of the sum of responses to singlewhisker stimulation.

For the data analysis of the stimulus specificity of the facilitatory response interaction, responses (samples) were classified into three categories: "selective", in which facilitatory interaction was observed for only one stimulus condition between two combinations of whiskers or between two directions of whisker deflection; "nonselective", in which facilitation was observed for both stimulus conditions; and "none", in which facilitation was not observed at all.

To examine whether direction-selective response facilitation is attributable to the direction preference of a single whisker response, we calculated the direction selectivity index (DI) for the responses to single and combined whisker stimulations according to the following formula:

$$
D I=(R r-R c) /(R r+R c),
$$

where $R r$ and $R c$ are the numbers of spikes elicited by whisker deflection in rostral and caudal directions, respectively. The DIs calculated for the sum of responses to single whisker stimulation of PW and AW and for the maximal response to multiwhisker stimulation were defined as DI (PW + AW) and DI (M), respectively.

To investigate the relationship between locations of recorded cells in relation to barrel columns and the pattern and magnitude of response interaction, cells were categorized into three groups (see Results), and a one-way ANOVA was performed for comparisons among the groups.

Histology. At the end of each penetration, dye marks were produced at the recording sites by passing tip-negative currents (intensity, $5 \mu \mathrm{A}$; duration, $1 \mathrm{sec}$ at $0.5 \mathrm{~Hz} ; 200$ pulses). After the recording experiment, the animals were deeply anesthetized with an overdose of anesthetics and perfused transcardially with PBS followed by $4 \%$ paraformaldehyde in phosphate buffer (PB). The recorded hemispheres of the cortex were flattened and post-fixed in $4 \%$ paraformaldehyde/30\% sucrose in PB for 4-12 hr. Sixty-micrometer-thick frozen tangential sections of the SI were prepared on a microtome and kept in PBS. Serial sections were histochemically stained for cytochrome oxidase (CO) (Wong-Riley, 1979). Then, the laminar locations of the recording sites and barrels in layer IV were identified by observation under a microscope. Finally, locations of cells in relation to the barrel columns were reconstructed by camera lucida drawing. For this analysis, images of tangential sections were superimposed by aligning with the reference holes of blood vessels. Because Nissl staining was not used, barrel territories were divided into two regions: the COdense centers (barrel hollow) and the $\mathrm{CO}$-sparse septal regions between 


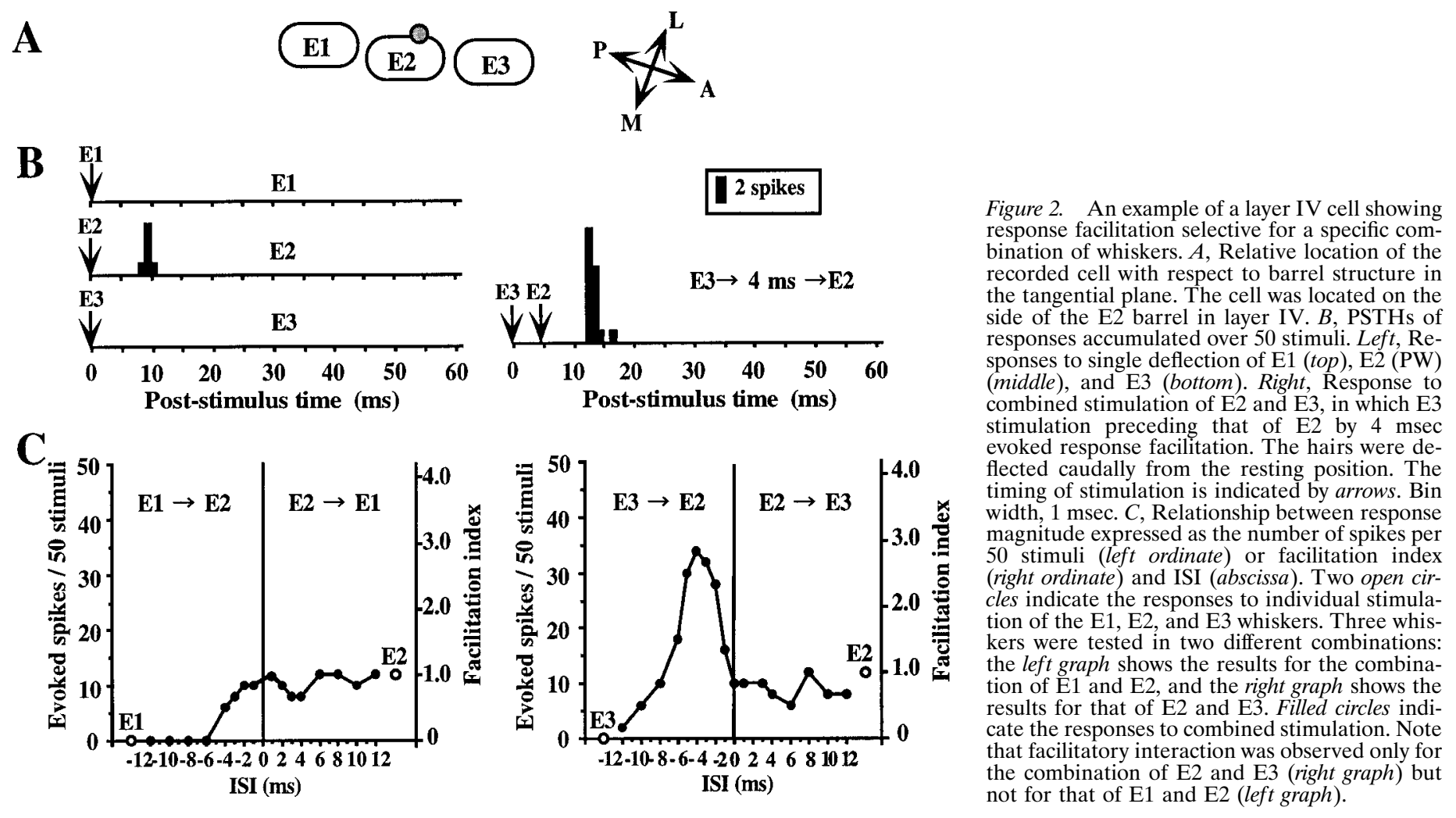

hollows (septa). Accordingly, the term "septa" used in this report includes both the barrel sides and the septa. These categories were also applied to other layers, taking account of their location relative to the barrel hollow or septa of layer IV.

\section{RESULTS}

Well isolated single-unit activity of 114 cells (layer II/III, $n=39$; $\mathrm{IV}, n=33$; V/VI, $n=42$ ) was recorded from the barrel cortex. Significant response facilitation $(\mathrm{FI} \geq 1.25)$ was observed in $37 \%$ of the cells analyzed.

\section{Selectivity for neighboring whiskers}

To examine whether there are specific combinations of neighboring whiskers that induce response facilitation, we tested the effects of different combinations of two whiskers. Figure 2 demonstrates a neuron representing typical combination-specific response facilitation. This cell was recorded in layer IV on the side of the E2 barrel close to the E3 barrel (Fig. 2A). All whiskers were deflected caudally from the resting position. When a single whisker was deflected, the cell responded only to deflection of the E2 whisker, but not to that of others (Fig. 2B, left PSTHs). However, when the deflection of the E3 whisker preceded that of the E2 whisker by 2-6 msec, a significant response facilitation (150-283\%) was noted (Fig. 2B, right PSTH, C, right graph). On the other hand, no response facilitation was observed when the E1 whisker was deflected in combination with E2 whisker (Fig. 2C, left graph). Moreover, when the deflection of E1 or E3 preceded that of E2 by $>6$ msec (for E1) or $10 \mathrm{msec}$ (for E3), the E2 response was strongly suppressed (Fig. 2C). This suggests that stimulation of the individual AWs would have evoked different subthreshold responses.

Results of the population analysis of the data from the combination-selectivity experiments are summarized in Figure 3. Because the induction of response facilitation often showed dependence on the direction of whisker deflection, we discriminated between two sets of results for each cell obtained using the same whisker combination but with different directions of whisker deflection, and called each set of data a sample. In total, 75 samples of 46 cells tested with two different kinds of combined stimulation using three whiskers, that is, one PW and either a rostral or a caudal AW, were analyzed for all the layers. Response facilitation
A

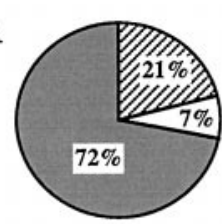

All layers

(46 cells, 75 samples)

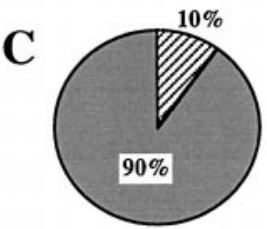

layer IV

(18 cells, 31 samples)

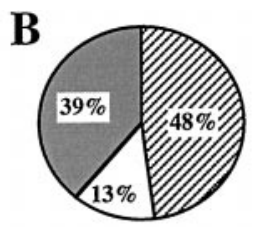

layer II/III

(16 cells, 23 samples)

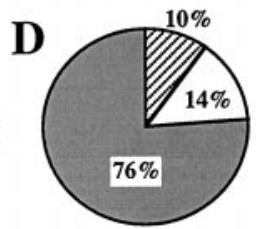

layer V/VI

(12 cells, 21 samples)

Figure 3. Population analysis of combination selectivity. The specificity of the response interaction was categorized into three classes, selective, nonselective, and none. The definitions are given in Materials and Methods. Percentages of samples in each category are indicated. $A$, All data. $B$, Layer II/III. C, Layer IV. D, Layer V/VI.

was observed in $28 \%$ of all the sample ( 21 of 75 samples) (Fig. $3 A$ ). The facilitation was most frequently observed in samples of layer II/III cells $(61 \%, 14$ of 23 samples), and whisker selectivity was seen in $79 \%$ of the samples that exhibited facilitation (Fig. $3 B$ ). The response facilitation was rarely seen in the samples of layer IV cells (10\%, 3 of 31 samples) (Fig. 3C) and was intermediate in those of layer V/VI cells (24\%, 5 of 21 samples) (Fig. $3 D$ ). In the layers IV and V/VI, whisker selectivity was seen in $63 \%$ of the samples that 
$\mathbf{A}$

Figure 4. Example of a layer II/III cell showing direction-selective facilitation. $A$, The cell was located on the side of the E2 barrel column. $B$, PSTHs of responses to deflections of E2 and E3. PSTHs in the left column, Whiskers were deflected in the rostral direction. PSTHs in the right column, Whiskers were deflected in the caudal direction. Top and middle, Responses to single whisker stimulation of E2 and E3, respectively. Bottom, E3 stimulation preceded E2 stimulation by 2 msec. Facilitation (243\%) was observed for the response to caudal deflection. Other notations are as in Figure 2. Note that both facilitatory and suppressive interactions were direction selective, whereas responses to single whisker stimulation were almost the same for the two directions (open circles in graphs, and $B$, middle and bottom).
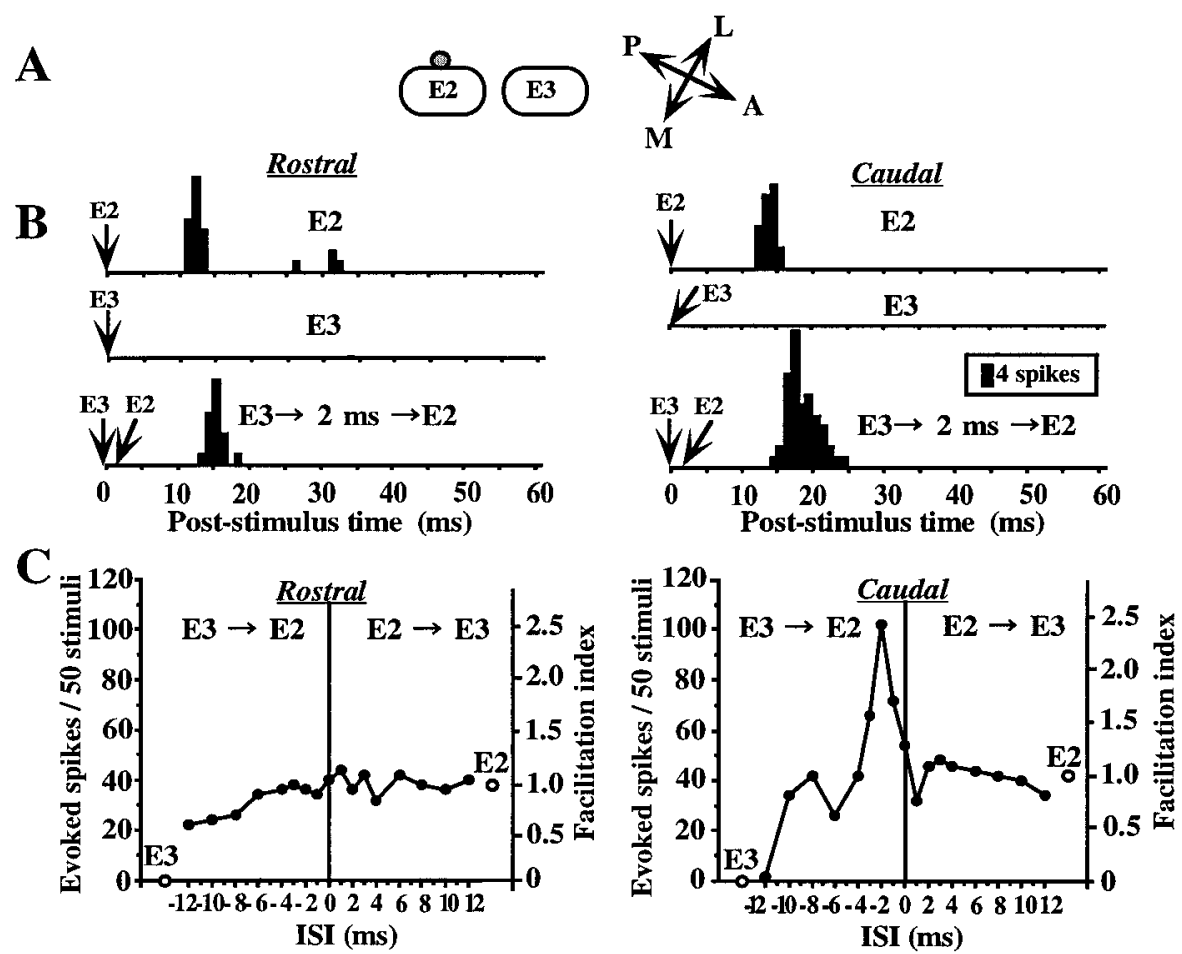

exhibited facilitation (Fig. 3C,D). Therefore, the facilitatory and whisker-selective interaction of response is a characteristic feature of cells in the superficial layers, even though it was observed to a lesser extent also in the other layers.

\section{Selectivity for direction of whisker deflection}

Cells in the barrel cortex are known to respond differentially to single whisker stimulation depending on the direction of whisker deflection (Simons, 1983, 1985). To examine the direction specificity for a facilitatory response interaction, we compared the responses to two different directions of whisker deflection, where whiskers were deflected either caudally or rostrally from the resting position. An example representing direction-selective response facilitation is shown in Figure 4. This cell was located on a side of the E2 barrel column in layer II/III (Fig. $4 A$ ). Single stimulation of the E2 whisker, but not of the E3 one, evoked an almost equivalent number of spike discharges, for both directions of deflection (Fig. $4 B$, top and middle PSTHs). However, in combined stimulation trials, a definite response facilitation (155-243\%) was observed when the E3 whisker was antecedently deflected caudally from the resting position when the ISI was set to $1-3 \mathrm{msec}$ (Fig. $4 B$, bottom right PSTH, C, right graph). In contrast, there was no facilitatory interaction when both whiskers were deflected in the opposite direction (Fig. 4B, bottom left PSTH, C, left graph). An inhibitory influence of the E3 whisker deflection on the response to E2 whisker deflection was also observed when both whiskers were deflected caudally for an ISI of $12 \mathrm{msec}$ (Fig. 4C, right graph). On the other hand, when the whiskers were deflected rostrally, antecedent deflection of E3, with the ISI still set at $12 \mathrm{msec}$, induced neither facilitation nor suppression (Fig. 4C, left graph). Thus, this cell clearly exhibited direction selectivity for both facilitatory and suppressive response interactions only when the whiskers were successively stimulated and did not for individual responses to single whisker stimulation.

Figure 5 shows an example representing a non-directionselective response facilitation, that is, bidirectional facilitation. The cell was recorded from layer II/III above the medial part of the septum of the E2 column (Fig. 5A). Stimulation of the E2 whisker evoked spike responses for either direction of whisker deflection (Fig. 5B, middle PSTHs), and that of the E1 whisker induced only negligible responses for both directions of deflection (Fig. 5B, top
PSTHs). Combined stimulation of the both E1 and E2 whiskers evoked response facilitation for both directions of deflection, when the two whiskers were stimulated at short ISIs (ISI $<4 \mathrm{msec}$ ) (Fig. $5 B$, bottom PSTHs, C, graphs).

The results of the experiments to determine the direction selectivity of the facilitatory interaction are summarized in Figure 6. In total, 97 samples from 64 cells were analyzed according to their laminar locations. The facilitatory interaction was most frequently observed for cells in layer II/III (50\%, 14 of 28 samples) regardless of the directionality. Moreover, more than half of the samples of cells in the layer II/III exhibiting response facilitation exhibited direction selectivity (57\%, 8 of 14 samples). Direction-specific facilitation was also observed in other layers but to a lesser extent (Fig. 6C,D). At the population level, there seemed to be no bias toward a particular direction of whisker deflection for inducing either bidirectional facilitation or direction-selective facilitation. The incidence of unidirectional response facilitation was similar for both the deflection directions. Among the 12 samples in which unidirectional facilitation was observed, the whisker deflection in the rostral direction induced facilitation in six samples, whereas that in the caudal direction in another six samples.

To examine the possibility that direction-selective response facilitation is attributable to the direction preference of a single whisker response, we calculated the DIs (see Materials and Methods) for the samples showing response facilitation. DIs for the multiwhisker responses (DI (M)) were plotted against those for the sum of the responses to single whisker stimulation of PW and AW $(\mathrm{DI}(\mathrm{PW}+\mathrm{AW}))$ in Figure 7. A linear relationship was noted between the two DIs $(r=0.83 ; p<0.001)$, and the slope of the regression line was almost equal to 1 . These results suggest that direction selectivity for response facilitation depends on a directional bias in the sum of the excitatory inputs derived from the two different whiskers.

\section{Dependence of response interaction on the location of neurons}

The induction and magnitude of facilitation depended on the cell position in relation to the barrel columns. We analyzed this point in detail using neurons recorded from the superficial layers for which facilitation was observed most frequently (Fig. 8). For this analysis, we chose particular cases in which whiskers were deflected 


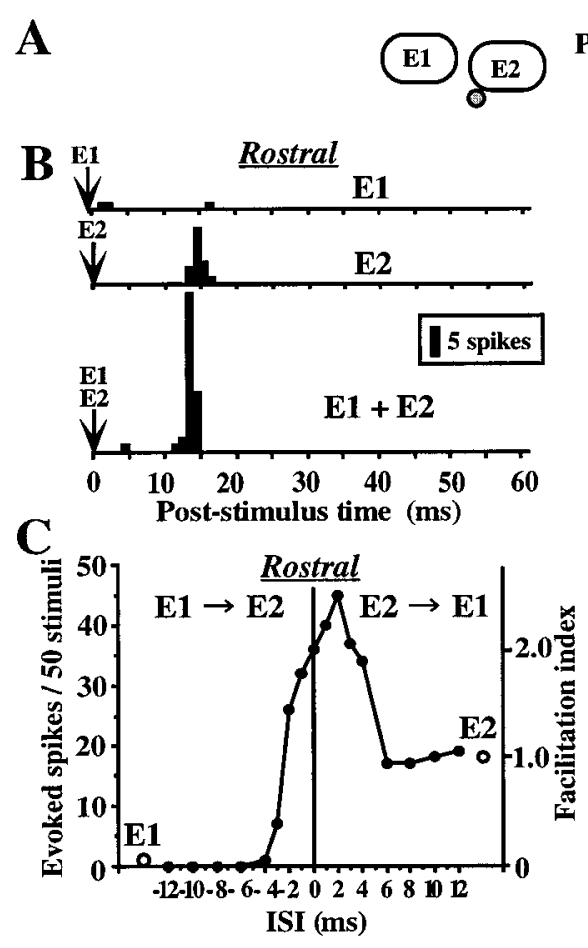

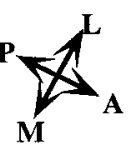
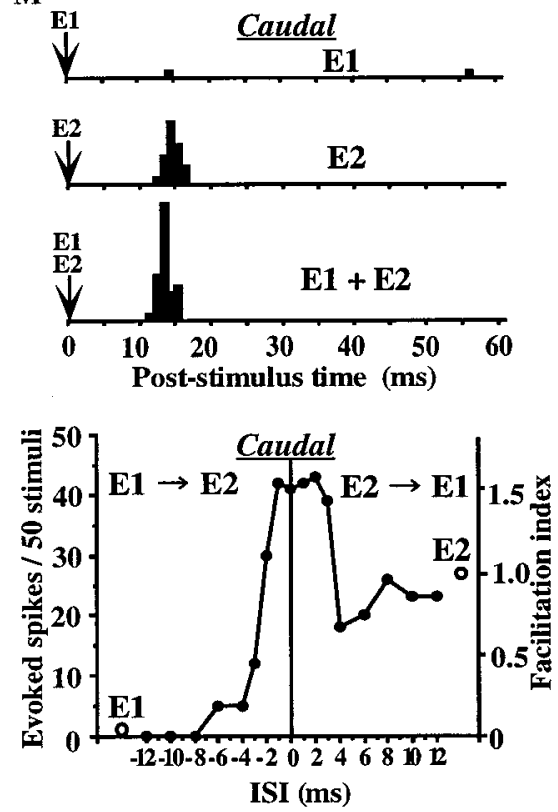

Figure 5. Example of a layer II/III cell showing nondirection-selective facilitation. $A$, The cell was located at the septal region near E2 barrel column. B, Left and right columns, Whiskers were deflected in the rostral (left) and caudal (right) directions. Top and middle, Responses to single whisker stimulation of E1 and E2, respectively. Bottom, Response to simultaneous stimulation of E1 and E2. Similar magnitudes of responses were observed for both directions of deflections. $C$, ISI tuning curve. Other notations are as in Figure 2.
A

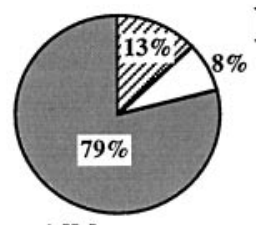

All layers

(64 cells, 97 samples)

C

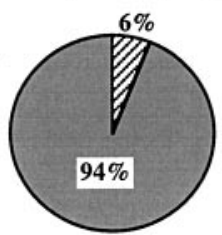

layer IV

( 22 cells, 36 samples)

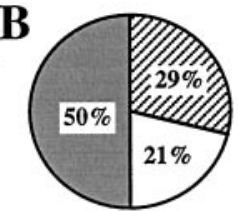

layer II/III

(17 cells, 28 samples)

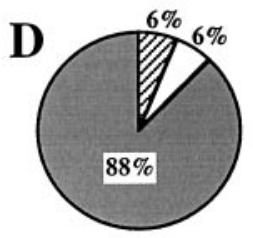

layer V/VI

( 25 cells, 33 samples)

\section{WII Selective (Unidirectional) \\ Nonselective (Bidirectional) \\ None}

Figure 6. Population analysis of direction selectivity. The specificity of the response interaction was categorized into three classes, selective, nonselective, and none. The definitions are given in Materials and Methods. Percentages of each category are indicated. $A$, All data. $B$, Layer II/III. $C$, Layer IV. $D$, Layer V/VI.

in the caudal direction, because for the cell group tested by whisker deflection in the rostral direction, the cells were not distributed randomly over the barrel columns, which is, therefore, irrelevant to this analysis. Also, fast-spiking units (FSUs) were excluded from this analysis, because FSUs showed no response facilitation (Shimegi et al., 1999). Figure 8, $A$ and $B$, shows microphotographs of CO-stained barrel structures in layer IV and a deposit of Pontamine Sky Blue (Fig. 8B, arrow) showing the recording site in layer II/III, respectively. Figure $8 C$ is the histological reconstruction of the recording site by superimposing the image of Figure $8 B$ on $A$; the cell is in the $\mathrm{E} 2$ barrel column and nearer to the $\mathrm{E} 3$ barrel

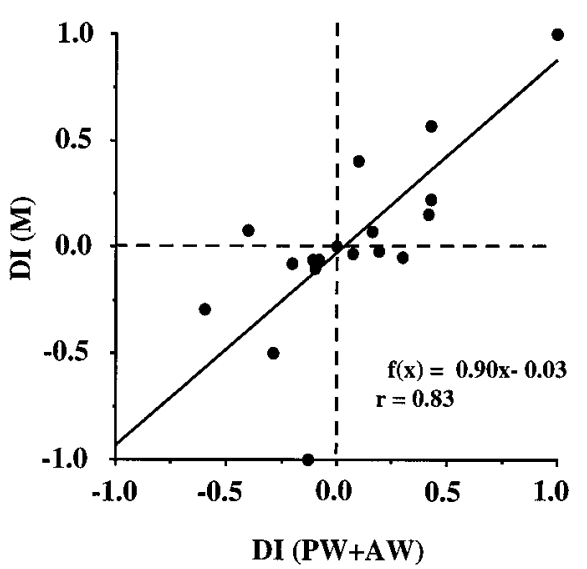

Figure 7. Relationship between DIs for multiwhisker response (DI $(\mathrm{PW}+\mathrm{AW}))$ and for the sum of single whisker responses (DI $(M))$. Data were obtained from all samples exhibiting response facilitation $(n=20)$. Regression line: $f(x)=0.90 x-0.03$. Correlation coefficient: $r=0.83(p<$ $0.001)$.

column than to the E1, as indicated by the arrow. Figure $8 D$ shows tangentially reconstructed locations of all cells analyzed in relation to the two barrel columns. Each dot indicates the location of one recorded cell. The cells were divided into three groups, that is, the rostral, middle, and caudal field groups. The middle field group consisted of cells located within the adjoining one-third of two barrels, and their septal area as shown in Figure $8 D$. The averaged FIs of each group were plotted against ISIs in Figure $8 E$ (mean \pm $1 \mathrm{SE}$ ). Positive values of ISI indicate that the rostral whisker was stimulated first, then the caudal one, and negative values indicate whisker stimulation in the reverse order.

Figure $8 E$ clearly shows that each group has a distinct characteristic of the averaged ISI tuning curve. A one-way ANOVA was performed for comparisons among the groups. Statistically significant differences $(p<0.05)$ were observed at short ISIs $(0$ and -1 $\mathrm{msec}$ ) and long ISIs (less than or equal to $-6 \mathrm{msec}$ and $>6 \mathrm{msec}$ ). Response facilitation was strongest for cells in the middle field group (open circles) when the ISI was equal to or shorter than 3 msec. The cells of this group probably received inputs from rostral 
A
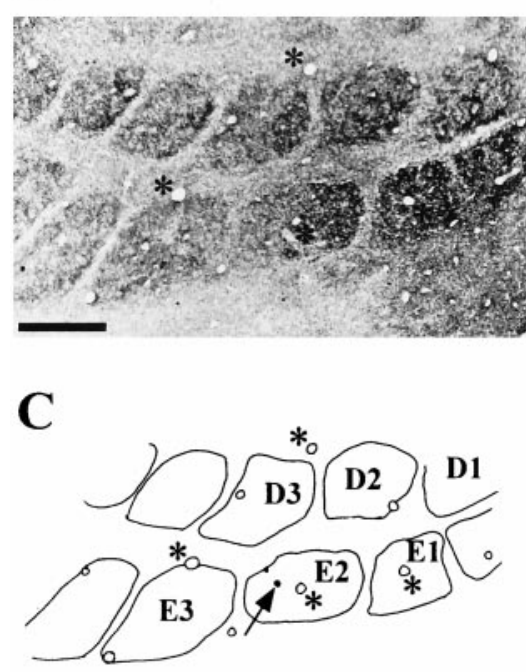

E

Figure 8. Relationship between response interaction and cell location in relation to the barrel structure in the superficial layers. $A, B$, Tangential sections of layer IV showing barrels $(A)$ and layer II/III $(B)$ of the PMBSF of a recorded animal. The section was stained for cytochrome oxidase. Arrow and asterisks indicate a dye mark of Pontamine Sky Blue produced at the recording site and blood vessels used as references for the reconstruction, respectively. Scale bar, 500 $\mu \mathrm{m} . C$, Recording site and barrel arrangement reconstructed by superimposing the image of $B$ on that of $A$. $D$, Tangential reconstruction of the distribution of cells. The barrels in layer IV are superimposed in dashed lines. Cells were divided into three groups based on the location in relation to two barrel columns; "caudal", "middle", and "rostral" field groups. Middle field group consisted of cells located adjacent to one-third of two barrels and the septal area. E, Averaged facilitation indices of each group for various ISIs (mean $\pm \mathrm{SE}$ ). Asterisks below the ISIs mean that there are statistically significant differences among the groups (one-way ANOVA, $p<0.05$ ).

and caudal whiskers with little difference in latencies. In the middle field group, when the ISI was $\geq 4 \mathrm{msec}$, the response interaction became weakly suppressive rather than facilitatory.

In contrast, the caudal field group ( filled circles) exhibited weak response facilitation when the rostral whisker was stimulated 1-3 msec before the caudal one. The cells of this group probably received the AW-related inputs first, before the PW-related inputs. When the ISI was increased to more than $6 \mathrm{msec}$, a strong suppression occurred.

These results suggest that excitation evoked in the barrel column by stimulation of the PW propagates well to the surrounding septal areas with a short $(\leq 3 \mathrm{msec})$ latency but only weakly into the central area of neighboring columns. However, the suppressive influence seems to extend far beyond the central area of the neighboring columns.

The response suppression was also observed in the rostral field group (open circles) when the AW stimulation preceded the PW stimulation at ISIs of $>4$ msec. There was a lack of response interaction in the cells of both rostral and caudal field groups when the corresponding PW was stimulated first, probably because the input from secondly stimulated AW with longer latency could not interact with the preceding excitation evoked by PW stimulation. The characteristic features of ISI tuning curves of individual groups, that is, the strong response facilitation of the middle field group for short ISIs and the strong response suppression of the caudal and the rostral groups for long ISIs were also observed after whisker deflection in the rostral direction, whereas the differences among the groups were not statistically significant because of insufficient data and sampling bias among the groups (data not shown).

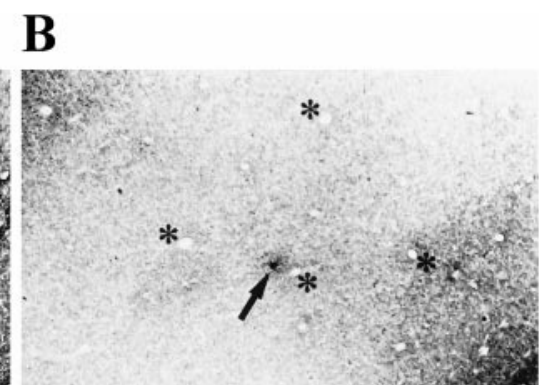

D

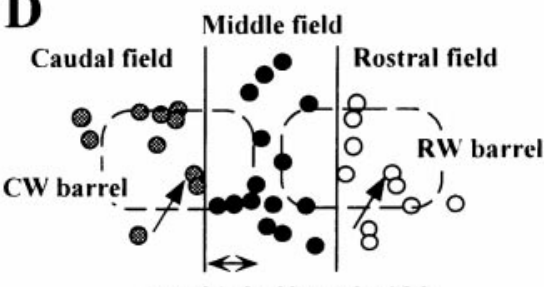

one third of barrel width

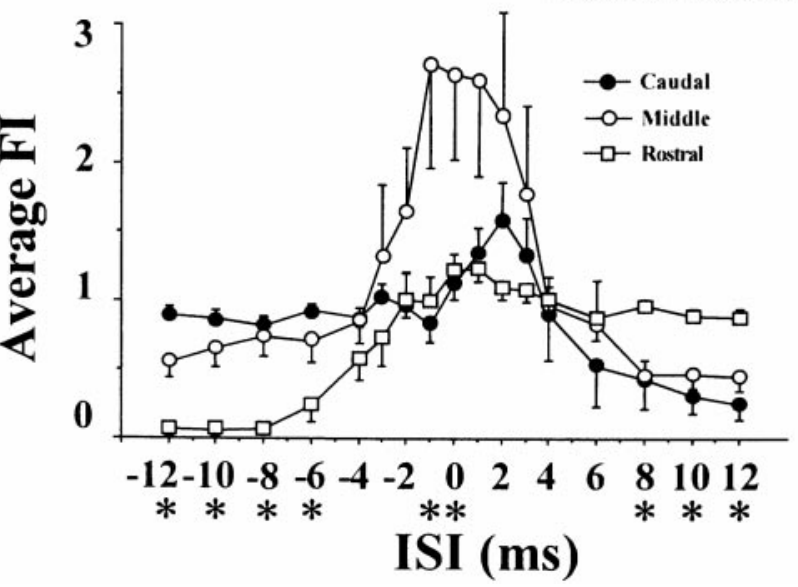

\section{DISCUSSION}

In the present study, we examined the stimulus specificity and the anatomical organization of response facilitation. The incidence and magnitude of response facilitation were strongly dependent on the particular combination of whiskers stimulated and the angular direction of whisker deflection. Moreover, the pattern and magnitude of response interaction induced by multiwhisker stimulation was closely related to the location of the cells in the tangential arrangement of barrel columns.

\section{Selectivity of response facilitation for a whisker combination}

In the majority of instances (75\%, 16 of 21 samples) in which response facilitation was observed, selectivity was noted for the combined stimulation of the PW with a particular AW in the same row (Fig. 3A). Therefore, response facilitation is thought to reflect the tactile events evoked after stimulation of the PW and a particular AW. Given that different combinations of whiskers successively come in contact with an object near the face of a rat, the combination-selective response facilitation would be able to provide a very fine spatial resolution for the detection of the characteristics of the object such as shape, surface structure, corner, edge, and speed and direction of motion.

Recent studies (Moore and Nelson, 1998; Zhu and Connors, 1999) reported that a subthreshold input from a single whisker is transmitted to many rows and arcs of cortical barrel columns. Therefore, subthreshold excitatory influence may arise even from remote barrel columns to a varying extent. In our study, however, the response facilitation was observed for responses to the stimulation of only a particular combination of neighboring whiskers in 
the majority of instances, even though we tested whisker combinations only within a row. It is known that the position of a cell in a barrel with respect to adjacent barrels is closely correlated with the magnitude and latency of its responses to stimulation of the corresponding surrounding whiskers (Armstrong-James et al., 1992; Welker et al., 1993). For example, if a cell located in the E2 barrel is closer to the E1 barrel than to the E3 barrel, a larger excitation is expected to be elicited by stimulation of the E1 whisker than that of the E3 whisker. Such a simple relationship between the magnitude of the excitatory influence and the anatomical proximity of the cell to neighboring barrels could be the basis for the whisker combination selectivity of response facilitation. This is consistent with our results that response facilitation to combined stimulation of a PW and an AW, whose corresponding barrel column is nearest to the recorded cell, was most prominent in cells in the superficial layers. On the other hand, layer V cells exhibit response facilitation to combined stimulation of a more than two whiskers (Ghazanfar and Nicolelis, 1997), because these possess a larger size of receptive fields than other layers (Chapin, 1986; Ghazanfar and Nicolelis, 1999).

\section{Possible input mechanisms for response facilitation}

The possible input mechanism underlying facilitatory response interaction in the superficial layers can be explained as follows. The whisker-derived information first relayed to the layer IV barrel is basically transmitted upward to layer II/III within the home barrel column in addition to the direct afferent inputs to layer III (White, 1978), then toward the superficial layers of adjacent columns (Connors, 1984; Armstrong-James et al., 1992; Kim and Ebner, 1999; Laaris et al., 2000). Therefore, cells in superficial layers can receive inputs not only from layer IV cells of the home barrel and afferents but also from layer II/III cells of adjacent columns via horizontal axonal projections (Bernardo et al., 1990; Connors and Amitai, 1993; Hoeflinger et al., 1995; Kim and Ebner, 1999; Laaris et al., 2000). In our study, cells located between two neighboring barrelcolumns (Fig. 8, middle field group) exhibited response facilitation for sequential stimulation with an ISI of $<3 \mathrm{msec}$ (Fig. $8 E$ ). Two excitations applied to each somatotopically corresponding barrel are transmitted to layer II/III, then these excitations arrive at the recorded cell at almost the same time. This could be the reason for the facilitation occurring when two whiskers are deflected at a very close interval.

However, for the cells at the caudal and rostral fields shown in Figure 8 , when the AW stimulation preceded the PW by a time corresponding to a difference of response latency (1-3 msec), the response facilitation was observed only to a lesser extent (Fig. 8E, caudal field group at positive ISIs) or was absent (Fig. $8 E$, rostral field group at negative ISIs). This implies that a propagation of excitation to neighboring barrel columns with sufficient strength to induce response facilitation are predominantly restricted to the septal region between the home and the adjacent columns, whereas the inhibitory influence prevails further with longer latency than the excitation.

We recently reported that there are two groups of layer V/VI cells that have different optimal ISI, that is, either short ( $\leq 3 \mathrm{msec})$ or long $(10-30 \mathrm{msec})$, with respect to the response facilitation (Shimegi et al., 1999). Layer V cells are known to receive inputs from all cortical layers, via their extensive basal and apical dendrites (Killackey et al., 1989; Koralek et al., 1990) in addition to direct afferent inputs from the thalamus to layer $\mathrm{Vb}(\mathrm{Lu}$ and $\mathrm{Lin}$, 1993). The short optimal ISI of the layer V cells may depend more on the afferent inputs, whereas the long optimal ISI, on the convergence of AW inputs of intracortical origin with longer latencies. It is also possible that the response facilitation of layer $\mathrm{V}$ simply reflects the augmented responses in VPM (Ghazanfar and Nicolelis, 1997).

Zhu and Connors (1999) reported that active and regenerative dendritic conductances amplify sensory signals in the barrel cortex in vivo. Moreover, Yoshimura et al. (2000) reported that inputs via horizontal axon collaterals to the pyramidal cells in the superficial layer of the cat visual cortex facilitatory interacted with inputs from layer IV in a voltage-dependent manner. Therefore, it is possible that intrinsic membrane properties of cortical neurons facilitate response augmentation to multiwhisker stimulation.

Our experiments were performed using anesthetized animals. However, recent study by Fanselow and Nicolelis (1999) reported that, in awake animals, tactile responses of cells in VPM and PMBSF were strongly modulated depending on the behavioral state of whisker movement. Even though the mechanisms of this modulation have not been clarified yet, the neuronal activity of somatosensory systems are more dynamically controlled to optimize tactile information processing according to the behavioral context under awake conditions.

\section{Direction selectivity}

In the present study, more than half of the response facilitation $(60 \%)$ occurred in a direction-specific manner for whisker deflection. The direction-selective response is thought to play a crucial role in detecting the direction of movements of nearby objects and the animal itself. It has been reported that the direction-selective response in the visual cortex is based on a linear summation of directionally biased excitatory and inhibitory inputs (Sato et al., 1995). In the barrel cortex, single whisker response is also reported to exhibit direction preference (Simons 1983, 1985). Therefore, by analogy with the visual cortex, directionally biased single whisker response to stimulation of either PW and AW could be a possible mechanism for the direction selectivity of response facilitation. We examined this point by calculating the DIs for the responses to single whisker and multiwhisker stimulations. The DIs, calculated as the linear sum of the two single responses to PW and AW stimulation, were clearly correlated with those calculated for the response to multiwhisker stimulation (Fig. 7). Therefore, directional bias of the single whisker response is likely to be one of the mechanisms underlying the direction preference of the multiwhisker responses. However, we also observed cases in which we could not predict the direction preference of multiwhisker response from that of two single whisker responses (Fig. 4). In such cases, a directional bias could exist in the subthreshold response to single whisker deflection.

\section{The role of facilitatory and inhibitory interaction of response}

The somatosensory system of rodents is well developed for regulating behavior in a narrow space. Although each of the whiskers is a discrete sensor for separate points of the surrounding space around the face, the spatiotemporal response interaction in the barrel cortex seems to enable an animal to represent his surrounding environment as a continuous space map with fine spatiotemporal resolution. We propose that an important role of response facilitation in the barrel cortex is to translate point-by-point information to a continuous space. Our hypothesis of the functional role of the response facilitation in the behavioral context is summarized as follows. During exploration of its environment by active whisker palpation, the animal changes its head direction along the surfaces of objects when vibrissal hairs move across the surface continually. Because whisking is precisely coordinated with movements of the nose, head, and body (Welker, 1964), the animal perceives the positions of the distal ends of individual vibrissae. Then it perceives the object from the point of whisker contact (Carvell and Simons, 1990). If only one whisker touched the object's surface, only a point position of the object, as determined by the deflection angle would be perceived. However, if two whiskers come in contact with the object simultaneously or at an ISI of within a few $(\sim 3 \mathrm{msec})$ milliseconds, information about the surface plane or surface orientation of the object would be expressed by the distribution of the response facilitation ("AND" response) in the superficial layers of the barrel cortex. Therefore, the animal should be able to determine the position, distance, and angle of the surface in relation to his face via the integration mechanism of the barrel cortex. 


\section{REFERENCES}

Armstrong-James M, Fox K, Das-Gupta A (1992) Flow of excitation within rat barrel cortex on striking a single vibrissa. J Neurophysiol 68:1345-1358.

Bernardo KL, McCasland JS, Woolsey TA, Strominger RN (1990) Loca intra- and interlaminar connections in mouse barrel cortex. J Comp Neurol 291:231-255.

Carvell GE, Simons DJ (1988) Membrane potential changes in rat SmI cortical neurons evoked by controlled stimulation of mystacial vibrissae. Brain Res 448:186-191.

Carvell GE, Simons DJ (1990) Biometric analyses of vibrissal tactile discrimination in the rat. J Neurosci 10:2638-2648.

Chapin JK (1986) Laminar differences in sizes, shapes, and response profiles of cutaneous receptive fields in the rat SI cortex. Exp Brain Res 62:549-559.

Chmielowska J, Kossut M, Chmielowski M (1986) Single vibrissal cortical column in the mouse labeled with 2-deoxyglucose. Exp Brain Res 63:607-619.

Connors BW (1984) Initiation of synchronized neuronal bursting in neocortex. Nature 310:685-687.

Connors BW, Amitai Y (1993) Generation of epileptiform discharge by local circuits of neocortex. In: Epilepsy: models, mechanisms and concepts (Schwartzkroin P, ed), pp 388-423. New York: Oxford UP.

Fanselow EE, Nicolelis MAL (1999) Behavioral modulation of tactile responses in the rat somatosensory system. J Neurosci 19:7603-7616.

Ghazanfar AA, Nicolelis MAL (1997) Nonlinear processing of tactile information in the thalamocortical loop. J Neurophysiol 78:506-510.

Ghazanfar AA, Nicolelis MAL (1999) Spatiotemporal properties of layer V neurons of the rat primary somatosensory cortex. Cereb Cortex 9:348-361.

Goldreich D, Peterson BE, Merzenich MM (1998) Optical imaging and electrophysiology of rat barrel cortex. II. Responses to paired-vibrissa deflections. Cereb Cortex 8:184-192.

Griffiths W (1960) Responses of wild and domestic rats to forced swimming. Psychol Rev 6:39-49.

Hoeflinger BF, Bennett Clarke CA, Chiaia NL, Killackey HP, Rhoades RW (1995) Patterning of local intracortical projections within the vibrissae representation of rat primary somatosensory cortex. J Comp Neurol 354:551-563.

Ito $\mathrm{M}$ (1985) Processing of vibrissa sensory information within the rat neocortex. J Neurophysiol 54:479-490.

Ito M, Kawabata M, Shoji R (1979) Responses of vibrissa-sensitive cortical neurons in normal and prenatally X-irradiated rat. J Neurophysiol 42:1711-1726

Keller A (1995) Synaptic organization of the barrel cortex. In: The barrel cortex of rodents, Cereb Cortex, Vol 11 (Jones E, Diamond T, eds), pp 221-262. New York: Plenum.

Killackey HP, Koralek K-A, Chiaia NL, Rhoades RW (1989) Laminar and areal differences in the origin of the subcortical projection neurons of the rat somatosensory cortex. J Comp Neurol 282:428-445.

Kim U, Ebner FF (1999) Barrels and septa: Separate circuits in rat barrel field cortex. J Comp Neurol 408:489-505.

Kleinfeld D, Delaney KR (1996) Distributed representation of vibrissa movement in the upper layers of somatosensory cortex revealed with voltage-sensitive. J Comp Neurol 375:89-108.

Koralek K-A, Olavarria J, Killackey HP (1990) Areal and laminar organization of corticocortical projections in the rat somatosensory cortex. J Comp Neurol 299:133-150.

Laaris N, Carlson GC, Keller A (2000) Thalamic-evoked synaptic interactions in barrel cortex revealed by optical imaging. J Neurosci 20:1529-1537.

Lu SM, Lin RC (1993) Thalamic afferents of the rat barrel cortex: a lightand electron-microscopic study using Phaseolus vulgaris leucoagglutinin as an anterograde tracer. Somatosens Motil Res 10:1-16.

Moore CI, Nelson SB (1998) Spatio-temporal subthreshold receptive fields in the vibrissa representation of rat primary somatosensory cortex. J Neurophysiol 80:2882-2892.

Richter C (1957) On the phenomenon of sudden death in animals and man. Psychol Med 19:190-198.

Sato H, Katsuyama N, Tamura H, Hata Y, Tsumoto T (1995) Mechanisms underlying direction selectivity of neurons in the primary visual cortex of the macaque. J Neurophysiol 74:1382-1394.

Schiffman P, Lore R, Passafiume J, Neeb R (1970) Role of vibrissae for depth perception in the rat (rattus norvegicus). Anim Behav 18:290-292.

Shimegi S, Ichikawa T, Akasaki T, Sato H (1999) Temporal characteristics of response integration evoked by multiple whisker stimulation in the barrel cortex of rats. J Neurosci 19:10164-10175.

Simons DJ (1983) Multi-whisker stimulation and its effects on vibrissa units in rat SmI barrel cortex. Brain Res 276:178-182.

Simons DJ (1985) Temporal and spatial integration in the rat SI vibrissa cortex. J Neurophysiol 54:615-635.

Simons DJ, Woolsey TA (1979) Functional organization in mouse barrel cortex. Brain Res 165:327-332.

Vincent S (1912) The function of the vibrissae in the behavior of the white rat. Behav Monographs 1:7-85.

Welker WI (1964) Analysis of sniffing of the albino rat. Behavior 22:223-244.

Welker E, Armstrong-James M, Van der Loos H, Kraftsik R (1993) The mode of activation of a barrel column: response properties of single units in the somatosensory cortex of the mouse upon whisker deflection. Eur J Neurosci 5:691-712.

White EL (1978) identified neurons in mouse SmI cortex which are postsynaptic to thalamocortical axon terminals: a combined Golgielectron microscopic and degeneration study. J Comp Neurol 181:627-662.

Wong-Riley M (1979) Changes in the visual system of monocularly sutured or enucleated cats demonstrable with cytochrome oxidase histochemistry. Brain Res 171:11-28.

Yoshimura Y, Sato H, Imamura K, Watanabe Y (2000) Properties of horizontal and vertical inputs to pyramidal cells in the superficial layers of the cat visual cortex. 20:1931-1940.

Zhu JJ, Connors BW (1999) Intrinsic firing patterns and whisker-evoked synaptic responses of neurons in the rat barrel cortex. J Neurophysiol 81:1171-1183. 